\title{
Lutein exerts anti-inflammatory effects in patients with coronary artery disease.
}

Rosanna W S Chung, Per Leandersson, Anna K Lundberg and Lena J onasson

The self-archived version of this journal article is available at Linköping University Electronic Press:

http:// urn.kb.se/ resolve?urn=urn:nbn:se:liu:diva- 138450

N.B.: When citing this work, cite the original publication.

Chung, R. W S, Leandersson, P., Lundberg, A. K, J onasson, L., (2017), Lutein exerts anti-inflammatory effects in patients with coronary artery disease., Atherosclerosis, 262, 87-93.

https://dx.doi.org/ 10.1016/j.atherosclerosis.2017.05.008

Original publication available at:

https:// dx.doi.org/10.1016/j.atherosclerosis.2017.05.008

Copyright: Elsevier

http:// www.elsevier.com/

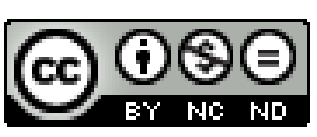




\begin{abstract}
Background and aims: Many coronary artery disease (CAD) patients exhibit chronic lowgrade inflammation. Carotenoids are anti-oxidants with potential anti-inflammatory properties. Here, we first assessed relationships between interleukin(IL)-6 and individual carotenoids in plasma from CAD patients. Based on the results, we proceeded to assess anti-inflammatory effects of one carotenoid, lutein, in peripheral blood mononuclear cells (PBMCs) from CAD patients.
\end{abstract}

Methods: Lutein+zeaxanthin (isomers with lutein being dominant), $\beta$-cryptoxanthin, lycopene, $\alpha$ - and $\beta$-carotene and IL-6 were measured in plasma from 134 patients with stable angina (SA) and 59 patients with acute coronary syndrome. In 42 patients, plasma measurements were also performed 3 months after coronary intervention. PBMCs from SA patients were pre-treated with lutein (1, 5 and $25 \mu \mathrm{M})$ for $24 \mathrm{~h}$ followed by $24 \mathrm{~h}$ incubation $+/$ lipopolysaccharide (LPS). Cell pellets were collected for IL-6, IL-1 $\beta$ and TNF mRNA and intracellular lutein. Cytokine secretion was measured in cell media.

Results: Only lutein+zeaxanthin were inversely correlated to IL-6 in SA patients at baseline $(r=-0.366, \mathrm{p}<0.001)$ and follow-up $(r=-0.546, \mathrm{p}<0.001)$. Ex vivo, lutein was taken up by PBMCs from SA patients in a dose- and time-dependent manner. Pre-treatment with lutein dosedependently lowered LPS-induced secretion of IL-6, IL-1 $\beta(p<0.01)$ and TNF $(p<0.05)$, and also reduced IL-6, IL-1 $\beta$ and TNF mRNA expression $(p<0.05)$.

Conclusions: Clinical findings highlighted the inverse association between lutein and IL-6 in CAD patients. Anti-inflammatory effects of lutein in PBMCs from CAD patients were consolidated in ex vivo experiments. Taken together, lutein has the potential to play a role in resolution of chronic inflammation in CAD patients.

Keywords: lutein; coronary artery disease; carotenoids; inflammation; cytokines; 


\section{Lutein exerts anti-inflammatory effects in patients with coronary artery disease}

Rosanna W.S. Chung ${ }^{1 *}$, Per Leanderson ${ }^{2}$, Anna K. Lundberg ${ }^{1}$, Lena Jonasson ${ }^{1}$

${ }^{1}$ Division of Cardiovascular Medicine, Department of Medical and Health Sciences, Linköping University, SE-581 85 Linköping, Sweden.

${ }^{2}$ Occupational and Environmental Medicine Center, Department of Clinical and Experimental Medicine, Linköping University, SE-581 83 Linköping, Sweden.

* Corresponding author: Rosanna W.S. Chung

Division of Cardiovascular Medicine

Department of Medical and Health Sciences

Faculty of Health Sciences

Linköping University

SE-581 85 Linköping

Sweden

Telephone: +46101037476

Fax: +4613145949

Email: Rosanna.chung@liu.se

Email addresses of co-authors:

Per Leanderson: per.leandersson@regionostergotland.se

Anna K. Lundberg: anna.lundberg@liu.se

Lena Jonasson: lena.jonasson@liu.se 


\section{Introduction}

Inflammation has a prominent role in coronary artery disease (CAD) and other manifestations of atherosclerosis, from the development of plaques to plaque destabilization [1]. Elevated inflammatory biomarkers in the circulation, like C-reactive protein (CRP) and interleukin (IL)6, have also been consistently shown to predict the risk of CAD [2, 3]. Over the last decades, significant improvements have been made in the medical and lifestyle management of CAD, all of which may lead to reductions in inflammatory activity. Despite this, low-grade systemic inflammation persists in many patients with CAD. There is growing evidence that antiinflammatory diets, such as Mediterranean diet, may have beneficial effects [4, 5]. However, the specific mechanisms linking diet to inflammation remain to be elucidated.

Carotenoids are lipid-soluble pigments found in a variety of fruits and vegetables. In several population-based studies, low plasma levels of carotenoids as well as low dietary intake of carotenoids have been associated with high cardiovascular disease risk [6, 7]. Moreover, inverse associations between circulating levels of carotenoids and inflammatory markers have been demonstrated in multiple studies [8-12] raising the hypothesis that carotenoids themselves exert anti-inflammatory effects. One might argue that individuals with low intake of carotenoid-rich food are more likely to have an overall unhealthy lifestyle since the association between carotenoids and inflammation has been shown to lose statistical significance after adjustment for potential confounders, such as overweight and smoking [13-17]. Moreover, majority of the mechanistic evidence were based on cell lines and animal models [7]. To the extent of our knowledge, it has not been investigated whether direct anti-inflammatory effects of carotenoids exist in human cells that are involved in the inflammatory response, such as peripheral blood mononuclear cells (PBMCs).

There are 6 major carotenoids in human plasma, lutein, zeaxanthine, $\beta$-cryptoxanthine, lycopene, $\alpha$-carotene and $\beta$-carotene. Lutein and zeaxanthine are stereoisomers with identical chemical formulas. Lutein is the predominant one with $\approx 5$-fold higher abundance in plasma $[18,19]$ and therefore the levels of lutein and zeaxanthine are commonly expressed as a combined figure. Although carotenoids share similar structural and chemical characteristics, there are evidence that they vary in biological effects. Previous studies that assess the effects of carotenoids on cardiovascular risk have focused mainly on $\beta$-carotene. Lately, lutein has emerged as a potentially stronger candidate for atheroprotection. A number of population-based studies comparing lutein with $\beta$-carotene have demonstrated that lutein, but not $\beta$-carotene, is 
associated with early protection against atherosclerosis as assessed by carotid intima-media thickness (IMT) [20-23]. A recent meta-analysis showed a lower risk of CAD and stroke if the highest and lowest tertiles of lutein blood levels or intake were compared [24]. Also, lutein has been shown to protect against atherosclerosis in animal models [20, 25, 26] and to exert antiinflammatory effects in various in vitro models [27, 28]. Moreover, recent studies in patients with early macular degeneration have shown that supplementation with lutein inhibits the systemic activation of the complement system [29, 30].

Comprehensive measurements of major carotenoids in plasma have been performed in population-based cohorts but are less common in patients with clinical manifestations of atherosclerotic disease. In a previous study, we found that CAD patients presenting with either acute coronary syndrome (ACS) or stable angina (SA) had significantly lower plasma levels of lutein+zeaxanthine compared with healthy controls, while levels of other major carotenoids, such as $\beta$-carotene and lycopene, did not differ between any subject groups [31]. In line with this, a larger case-control study on ACS reported that plasma levels of lutein were significantly lower in ACS patients compared with healthy controls while no significant differences were seen in $\beta$-carotene and lycopene [32].

In the present study, we first assessed the association between plasma levels of IL-6 and different carotenoids in patients with CAD. Using both a cross-sectional and longitudinal study design, we showed that IL-6 was inversely correlated with lutein+zeaxanthine but not with other major carotenoids in plasma at both baseline and follow-up. Since lutein is the dominant isomer, we proceeded to examine the anti-inflammatory effects of pure lutein on isolated PBMCs from patients with CAD and showed that lutein i) was stored in PBMCs and ii) suppressed their production of pro-inflammatory cytokines.

\section{Materials and methods}

\section{Subjects}

For plasma measurements of carotenoids and IL-6, 59 patients with recent onset of ACS (i.e. within $24 \mathrm{~h}$ after admission) and 134 patients with SA were consecutively recruited at the Department of Cardiology, University Hospital, Linköping, Sweden. Since IL-6 has a circadian rhythm, samples were always obtained in the morning. In order to avoid the inflammatory response to balloon injury and stent implantation, blood sampling was also performed prior to 
coronary angiography. ACS patients were included if they had a diagnosis of unstable angina/non-ST elevation myocardial infarction, with the diagnosis based on typical ECGchanges (ST-T segment depression and/or T-wave inversion) and/or elevated troponins. SA patients were included if they had an angiographically verified diagnosis of CAD and exhibited angina class I-III in accordance with Canadian Cardiovascular Society Classification without any worsening of symptoms in 3 months. Patients were excluded if they were older than 75 years, had severe heart failure, immunological disorders, neoplastic disease, evidence of acute or recent ( $<2$ months) infection, recent major trauma, surgery or revascularization procedure, treatment with immunosuppressive or anti-inflammatory agents (except low-dose aspirin). A longitudinal assessment was performed in 42 of the patients (14 ACS, 28 SA). In this subpopulation, blood samples were collected prior to coronary angiography and 3 months after coronary intervention. The reason that only 42 patients participated in the longitudinal study was mainly due to geographic or logistic factors. The study was conducted in accordance with the ethical guidelines of Declaration of Helsinki, and the research protocol was approved by the Ethical Review Board of Linköping University. Written informed consent was obtained from all study participants.

For ex vivo experiments, blood was obtained from patients with SA with similar criteria as above.

\section{Extraction of carotenoids}

Carotenoids in heparinized plasma $(150 \mu \mathrm{L})$ and PBMCs in $150 \mu \mathrm{L}$ PBS were extracted in duplicates using Milli-Q water, 95\% ethanol with $0.1 \%$ butylated hydroxytoluene and pure hexane in a ratio of 3:3:4 (v/v/v) in one round of extraction. In brief, water and ethanol were added to plasma or PBMCs suspended in PBS followed by vortex at full speed for a few seconds. Thereafter, hexane was added and samples were vortexed at $2400 \mathrm{rpm} / \mathrm{min}$ for $5 \mathrm{~min}$. At this point, proteins and carotenoids were dissolved in the ethanol-water phase and the hexane phase, respectively. The hexane phase was isolated by centrifugation at $14000 \mathrm{xg}$ for $2 \mathrm{~min}$. After centrifugation, the upper phase (hexane phase) was transferred to a new tube and dried under nitrogen gas. The carotenoids were then reconstituted in $100 \mu \mathrm{L}$ mobile phase $(80 \%$ acetonitrile:20 \% methanol) followed by 5 min sonication in a sonication water bath prior to high-performance liquid chromatography (HPLC) analysis. 


\section{Quantification of carotenoids}

Carotenoids (lutein, zeaxanthin, $\beta$-cryptoxanthin, lycopene, $\alpha$-carotene, $\beta$-carotene) levels in both plasma and PBMCs were determined by HPLC using a modified version as previously described [31]. As stereoisomers, lutein and zeaxanthin were measured together and presented as a combined value in plasma. The HPLC analyses were performed using a PU 980 HPLC Pump (Jasco Inc., Japan), C18-Chromolith ${ }^{\circledR}$ Performance RP-18 endcapped 100-4.6 HPLC column that has a $4.6 \mathrm{~mm}$ internal diameter packed with C18-Chromolith material and $2 \mu \mathrm{m}$ macropores (Merck KGaA, Germany) and Jasco MD-2010 Plus Multiwavelength Detector (Jasco Inc., Japan). The flow rate was set to $3 \mathrm{~mL} / \mathrm{min}$ and $20 \mu \mathrm{L}$ of samples were injected. Signals were recorded at $450 \mathrm{~nm}$ using Clarity version 2.6.5 (DataApex, Czech Republic). Carotenoid concentration were calculated from a standard curve generated from reference samples that were calibrated against an external standard (SRM 968E) from the National Institute of Standard and Technology. Inter-assay coefficient of variations were less than or equal to 5 .

\section{Cytokine measurements}

Levels of IL-6, IL-1 $\beta$ and tumor necrosis factor (TNF) were analyzed by high-sensitivity human multiple Luminex assay (Bio-Techne, UK). The sensitivity limits of quantification for IL-6, IL$1 \beta$ and TNF were 2.3-18880, 3.2-3261, and 5.8-95484 pg/mL, respectively. The interassay CVs for IL-6, IL-1 $\beta$ and TNF were $7 \%, 6 \%$ and $8 \%$, respectively.

\section{Gene expression analysis}

Total RNA was isolated from PBMCs using an RNeasy kit (Qiagen, Germany). Purity of RNA was tested in a 2100 Bioanalyzer (Agilent Technologies, USA). RNA (100 ng) was reverse transcribed into cDNA using High Capacity cDNA Reverse Transcription Kits (Applied Biosystems, USA). Glyceraldehyde 3-phosphate dehydrogenase (GAPDH, NM_001256799.2), IL-6 (NM_000600.4), TNF (NM_000594.3) and IL-1ß (MN_000576.2) mRNA were amplified using TaqMan Gene Expression Assays in a 7500 Fast Real-Time PCR System (Applied Biosystems, USA). PCR conditions were as described by the manufacturer. The relative gene expression was calculated by normalizing cycle threshold $(\mathrm{Ct})$ values for genes of interest with $\mathrm{Ct}$ values for GAPDH using the $\Delta \Delta \mathrm{Ct}$ method. 
In order to prevent oxidation of lutein during incubation, lutein was incorporated into micelles before being supplemented to PBMCs. The micellar cell medium solution was prepared by dissolving $33 \mu \mathrm{M}$ oleic acid, $16.7 \mu \mathrm{M}$ L- $\alpha$-phosphatidylcholine, $100 \mu \mathrm{M}$ monoolein and $2 \mathrm{mM}$ sodium taurocholate hydrate in complete cell medium via 15 min sonication in water bath followed by sterilization through a $0.22 \mu \mathrm{m}$ filter [33]. The complete cell medium contained Roswell Park Memorial Institute (RPMI) 1640 medium (Life Technologies, USA), 10 \% fetal bovine serum (FBS), 100U/mL Penicillin-Streptomycin (Life Technologies, USA). All other chemicals were purchased from Sigma Aldrich (USA) unless specified otherwise. To make lutein micellar cell medium, $1 \mu \mathrm{M}, 5 \mu \mathrm{M}$ or $25 \mu \mathrm{M}$ lutein analytical standard (07168, Sigma Aldrich, USA) was dissolved with all of the micelle components mentioned above in complete cell medium via the same sonication and sterilization procedures. The lutein analytical standards had $\geq 97 \%$ purity, according to certificates of analysis provided by Sigma Aldrich.

To confirm the concentration of lutein used in the ex vivo PBMC treatment, a known amount of lutein analytical standard was first reconstituted in absolute ethanol. Several diluted lutein samples were then prepared via serial dilution and their absorbance at $445 \mathrm{~nm}$ were measured in a spectrophotometer. The actual concentrations of the original lutein analytical standard was then determined using molar extinction coefficient of lutein in absolute ethanol $\left(\varepsilon=145 \times 10^{3}\right)$.

Treatment of PBMCs with lutein in the presence or absence of LPS

PBMCs were isolated from venous blood of patients with SA using Ficoll-Paque Plus (GE Healthcare Life Science, UK) according to manufacturer's instructions. PBMCs were incubated in the micellar cell medium containing $1 \mu \mathrm{M}, 5 \mu \mathrm{M}$ or $25 \mu \mathrm{M}$ lutein for 24 hours under standard cell culturing conditions before incubation with (or without) additional $1 \mathrm{ng} / \mathrm{mL}$ lipopolysaccharide (LPS) for 24 hours. Countess II Automated Cell Counter (Life Technologies, USA) was used to obtain cell concentrations as per manufacturer's instructions. Seeding density was 1000000 cells/mL. At the end of the incubation, cell media and cell pellets were collected and stored in $-80^{\circ} \mathrm{C}$ until analysis. CellTiter 96 aqueous one solution cell proliferation assay (MTS) (Promega, USA) was performed to assess viability of PBMCs, according to manufacturer's instruction. 
Statistical analysis

IBM SPSS Statistics 23 was used for statistical analyses. For all clinical and laboratory characteristics, the significance of any difference between SA and ACS patients was analyzed using Mann-Whitney U-test. Bivariate correlations were analysed by Spearman's rank correlation coefficient. A multiple linear regression analysis was performed to assess the independent contribution of different factors to lutein+zeaxanthin levels in plasma. Differences between related samples were analysed by Friedman's or Wilcoxon Sign rank tests. Two-tailed p-values $<0.05$ were considered significant.

\section{Results}

\section{Characteristics of study population}

The characteristics of patients with SA and ACS are listed in Table 1. There were no significant differences in age, gender, current smoking, waist circumference, hypertension or prior history of ACS between the two groups. Type 2 diabetes was more common in the ACS group. Also, the ACS patients had lower prevalence of statin use and higher levels of total cholesterol, LDL cholesterol and triglyceride levels compared with the SA patients. Moreover, the ACS group had significantly higher levels of plasma IL-6.

For ex vivo experiments, PBMCs were prepared from a total of 16 SA patients (25\% females, median age 65 years). All 16 patients were treated with high-dose statin, i.e. $80 \mathrm{mg}$ atorvastatin.

\section{Lutein+zeaxan8thin were inversely associated with IL-6 in plasma}

The cross-sectional associations between plasma carotenoids levels and inflammatory status, as assessed by IL-6, are shown in Table 2. Lutein+zeaxanthin levels were inversely correlated to IL-6 in the whole cohort $(r=-0.190, p<0.05)$ and in the SA group $(r=-0.366, p<0.001)$, but not in the ACS group. Other carotenoids did not show any correlations to IL-6. In the SA group, lutein+zeaxanthin levels were inversely correlated to age $(r=-0.262, p<0.01)$ and waist circumference $(r=-0.208, p<0.05)$. In a multiple regression model, age, waist circumference and IL-6 levels were included as independent variables and lutein+zeaxanthin levels as dependent variable. Lutein+zeaxanthin levels remained significantly associated with IL-6 levels (adjusted $\left.r^{2}=0.064, p<0.05\right)$. 
When plasma carotenoids and IL-6 were reassessed in 42 patients 3 months after a percutaneous coronary intervention procedure, the bivariate correlation analysis still showed a significant inverse correlation between lutein+zeaxanthin and IL-6 levels, $r=-0.546, p<0.001$.

Lutein was taken up by PBMCs from SA patients in a dose- and time-dependent manner

PBMCs from SA patients were treated with unloaded micelles or micelles loaded with lutein at different concentrations (1, 5 or $25 \mu \mathrm{M})$ in a standard cell culture setting. As shown in Figure 1A, the intracellular concentrations of lutein increased dose-dependently during a 24-hour incubation. Over 48 hours, the uptake of $25 \mu \mathrm{M}$ lutein by PBMCs also occurred in a timedependent manner (Figure 1B).

\section{Lutein suppressed cytokine production by PBMCs from SA patients}

To investigate the effect of lutein on LPS-stimulated cytokine production, PBMCs from SA patients were incubated with unloaded micelles or micelles loaded with lutein at different concentrations (1, 5 or $25 \mu \mathrm{M})$ for a total of 48 hours. As shown in Figure 2, the concentrations of IL-6, IL-1 $\beta$ and TNF in cell supernatants were dose-dependently reduced by lutein. We also investigated whether lutein affected gene expression of cytokines in PBMCs from SA patients and found that $25 \mu \mathrm{M}$ lutein suppressed LPS-induced mRNA expression of IL-6, IL1- $\beta$ and TNF (Figure 3). The cell viability, as assessed by the MTS assay, was not affected by the lutein treatment, nor by the LPS treatment (data not shown).

\section{Discussion}

In a clinical investigation of CAD patients, we found that lutein+zeaxanthine was the only type of carotenoid in plasma that correlated inversely with IL-6 at baseline (prior to coronary angiography) and at 3 months follow-up. We therefore proceeded to investigate the directacting effects of pure lutein on PBMCs from CAD patients and found that lutein suppressed both production and release of IL-6 and related cytokines.

Circulating levels of total and individual carotenoids, including lutein+zeaxanthine, have previously been shown to correlate inversely with slightly elevated markers of inflammation, i.e. low-grade systemic inflammation, in large population-based studies [8-11]. Fewer studies have examined the association between carotenoids and inflammatory markers in patients with chronic diseases. Also, most studies have focused on measuring CRP instead of IL-6. To our 
knowledge, there is only one study of elderly women showing that those with lowest carotenoid levels were more likely to have increasing IL-6 levels over a 2-year period [34]. Our rationale behind choosing IL-6 was that CRP is not considered the ideal marker of inflammation in individuals on statin treatment. Statins significantly reduce CRP levels in plasma, an effect that may be partly explained by prevention of IL-6-induced CRP expression in hepatocytes [35, 36]. On the other hand, statins has not been shown to affect IL-6 levels in plasma [37-39] and may therefore be more useful for assessing inflammation in patients with $\mathrm{CAD}$, at least under metabolic stable conditions. We did not see any relationship between lutein+zeaxanthine and IL-6 in patients with ACS, a condition associated with an acute-phase inflammatory response. Instead, the relationship between lutein+zeaxanthine and IL-6 was confined to the SA patient group, illustrating a potential role of lutein in low-grade chronic inflammatory conditions. The correlation also remained significant after adjustment for smoking and waist circumference. This is in line with a large population-based study of more than 7000 US inhabitants showing that lutein+zeaxanthine were inversely associated with CRP levels after adjustment for traditional cardiovascular risk factors [9].

The ex vivo results in the present study confirmed that lutein was capable of exerting antiinflammatory effects in human PBMCs. The administration of lutein to PBMCs, isolated from SA patients, dose-dependently led to intracellular accumulation of lutein and suppression of LPS-induced IL-1 $\beta$, TNF and IL-6 at both mRNA and protein levels. Others have examined anti-inflammatory effects of lutein in various cell lines, such as mouse macrophages and human colorectal adenocarcinoma cells. Pretreatment with lutein at doses between 10 and $20 \mu \mathrm{M}$ has been shown to prevent leukocyte adhesion and expression of inflammatory genes, e.g. TNF and IL-6, by inhibiting NF-кB signaling [40-42]. Also, parenteral administration of lutein in various

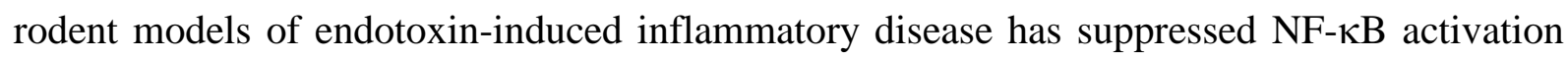
and inflammatory gene expression in various cells and tissues, including iris-ciliary body and peritoneal macrophages [40, 42]. Besides PBMCs, there are several potential sources of IL-6 in human plasma, including fibroblasts, endothelial cells, smooth muscle cells and adipocytes. Thus, it is reasonable to assume that lutein can affect circulating levels of IL-6 by suppressing cytokine production in other cell types besides PBMCs.

Population-based studies have consistently shown that higher levels of lutein are associated with decreased carotid IMT, raising the hypothesis that lutein plays a role in early protection against atherosclerosis [20-23]. This has been confirmed in animal models where lutein 
supplementation prevents the development of atherosclerotic lesions [20, 25, 26]. Atheroprotective effects have been attributed to both antioxidant and anti-inflammatory actions. The ability of lutein to prevent cell lipid oxidation and oxidative cell damage is well documented [7]. Dwyer et al [20] reported that lutein supplementation inhibited the inflammatory response of monocytes to oxidized LDL in atherosclerosis-prone mice. In a guinea pig model, a lutein-rich diet prevented atherosclerosis by lowering concentrations of oxidized LDL as well as decreasing malondialdehyde and cytokine production in aortic tissue [25]. In the present study, lutein was shown to suppress LPS-induced inflammatory gene expression in PBMCs, thus highlighting its anti-inflammatory properties.

It is worthwhile to note that we used PBMCs from patients with manifest CAD in the ex vivo experiments. This may be considered a strength since, in a secondary prevention perspective, it is important to verify that lutein is able to exert anti-inflammatory effects in PBMCs from patients who are on optimal medical therapy, including high-dose statin.

Some limitations of our study deserve consideration. The sample size was small and did not allow us to evaluate the impact of potential confounders like diabetes, obesity and health behaviors. The lack of food frequency questionnaires providing data on carotenoid intake may also be considered a limitation. However, the methodological problems associated with measurements of dietary intake are well-known. Also, in a previous study [14], no correlations between intake of carotenoid-rich fruits and vegetables and inflammatory markers were seen in a population-based cohort. Another limitation of the present study is the lack of a healthy control population. However, if a difference in correlates or effects of lutein had been observed between medicated patients and unmedicated healthy controls, it would have been difficult to interpret whether it represented the effect of CAD itself or the effect of medication. Furthermore, we only measured one of the cytokines (IL-6) in plasma since TNF and IL-1 $\beta$ cannot be measured reliably in clinical samples due to their pulsatile secretion pattern and short half-lives.

In conclusion, our data indicate that lutein by itself has the potential to exert significant antiinflammatory effects in patients with CAD, thus revealing one mechanism by which lutein can contribute to atheroprotection. Future dietary intervention studies are warranted to confirm whether increasing the consumption of lutein has beneficial effects on clinical outcomes in patients with CAD. 


\section{Conflict of interest}

There is no conflict of interest to be declared by any of the authors with respect to this manuscript.

\section{Acknowledgements}

This research was funded by the Heart Lung Foundation, Sweden (Grant number: 20150648) and Swedish Research Council, Sweden (Reference number: 2014-2479).

\section{Author contributions}

R.W.S. designed and performed the experiments and analyzed the data. R.W.S. and L.J. interpreted the data and wrote the manuscript. A.L. and P.L. provided scientific expertise and reviewed the manuscript.

\section{References}

[1] Libby P, Ridker PM, Hansson GK. Progress and challenges in translating the biology of atherosclerosis. Nature. 2011;473:317-25.

[2] Danesh J, Wheeler JG, Hirschfield GM, Eda S, Eiriksdottir G, Rumley A, et al. C-reactive protein and other circulating markers of inflammation in the prediction of coronary heart disease. N Engl J Med. 2004;350:1387-97.

[3] Kaptoge S, Seshasai SR, Gao P, Freitag DF, Butterworth AS, Borglykke A, et al. Inflammatory cytokines and risk of coronary heart disease: new prospective study and updated meta-analysis. Eur Heart J. 2014;35:578-89.

[4] Garcia-Arellano A, Ramallal R, Ruiz-Canela M, Salas-Salvado J, Corella D, Shivappa N, et al. Dietary Inflammatory Index and Incidence of Cardiovascular Disease in the PREDIMED Study. Nutrients. 2015;7:4124-38.

[5] Salas-Salvado J, Garcia-Arellano A, Estruch R, Marquez-Sandoval F, Corella D, Fiol M, et al. Components of the Mediterranean-type food pattern and serum inflammatory markers among patients at high risk for cardiovascular disease. Eur J Clin Nutr. 2008;62:651-9.

[6] Howard AN, Thurnham DI. Lutein and atherosclerosis: Belfast versus Toulouse revisited. Med Hypotheses. 2017;98:63-8.

[7] Pietro ND, Tomo PD, Pandolfi A. Carotenoids in cardiovascular disease prevention. JSM Atherosclerosis. 2016;1:1002.

[8] Ford ES, Liu S, Mannino DM, Giles WH, Smith SJ. C-reactive protein concentration and concentrations of blood vitamins, carotenoids, and selenium among United States adults. Eur J Clin Nutr. 2003;57:1157-63.

[9] Gruber M, Chappell R, Millen A, LaRowe T, Moeller SM, Iannaccone A, et al. Correlates of serum lutein + zeaxanthin: findings from the Third National Health and Nutrition Examination Survey. J Nutr. 2004;134:2387-94.

[10] Hozawa A, Jacobs DR, Jr., Steffes MW, Gross MD, Steffen LM, Lee DH. Relationships of circulating carotenoid concentrations with several markers of inflammation, oxidative stress, and endothelial dysfunction: the Coronary Artery Risk Development in Young Adults (CARDIA)/Young Adult Longitudinal Trends in Antioxidants (YALTA) study. Clin Chem. 2007;53:447-55.

[11] Kritchevsky SB, Bush AJ, Pahor M, Gross MD. Serum carotenoids and markers of inflammation in nonsmokers. Am J Epidemiol. 2000;152:1065-71.

[12] Thurnham DI, Northrop-Clewes CA. Inflammation and biomarkers of micronutrient status. Curr Opin Clin Nutr Metab Care. 2016;19:458-63.

[13] Rowley K, Walker KZ, Cohen J, Jenkins AJ, O'Neal D, Su Q, et al. Inflammation and vascular endothelial activation in an Aboriginal population: relationships to coronary disease risk factors and nutritional markers. Med J Aust. 2003;178:495-500. 
[14] Ryden M, Garvin P, Kristenson M, Leanderson P, Ernerudh J, Jonasson L. Provitamin A carotenoids are independently associated with matrix metalloproteinase-9 in plasma samples from a general population. J Intern Med. 2012;272:371-84.

[15] Suzuki K, Inoue T, Hashimoto S, Ochiai J, Kusuhara Y, Ito Y, et al. Association of serum carotenoids with high molecular weight adiponectin and inflammation markers among Japanese subjects. Clin Chim Acta. 2010;411:1330-4.

[16] van Herpen-Broekmans WM, Klopping-Ketelaars IA, Bots ML, Kluft C, Princen H, Hendriks HF, et al. Serum carotenoids and vitamins in relation to markers of endothelial function and inflammation. Eur J Epidemiol. 2004;19:915-21.

[17] Wang L, Gaziano JM, Norkus EP, Buring JE, Sesso HD. Associations of plasma carotenoids with risk factors and biomarkers related to cardiovascular disease in middle-aged and older women. Am J Clin Nutr. 2008;88:747-54.

[18] Handelman GJ, Nightingale ZD, Lichtenstein AH, Schaefer EJ, Blumberg JB. Lutein and zeaxanthin concentrations in plasma after dietary supplementation with egg yolk. Am J Clin Nutr. 1999;70:247-51.

[19] Brevik A, Andersen LF, Karlsen A, Trygg KU, Blomhoff R, Drevon CA. Six carotenoids in plasma used to assess recommended intake of fruits and vegetables in a controlled feeding study. Eur J Clin Nutr. 2004;58:1166-73.

[20] Dwyer JH, Navab M, Dwyer KM, Hassan K, Sun P, Shircore A, et al. Oxygenated carotenoid lutein and progression of early atherosclerosis: the Los Angeles atherosclerosis study. Circulation. 2001;103:2922-7.

[21] Dwyer JH, Paul-Labrador MJ, Fan J, Shircore AM, Merz CN, Dwyer KM. Progression of carotid intima-media thickness and plasma antioxidants: the Los Angeles Atherosclerosis Study. Arterioscler Thromb Vasc Biol. 2004;24:313-9.

[22] Iribarren C, Folsom AR, Jacobs DR, Jr., Gross MD, Belcher JD, Eckfeldt JH. Association of serum vitamin levels, LDL susceptibility to oxidation, and autoantibodies against MDA-LDL with carotid atherosclerosis. A case-control study. The ARIC Study Investigators. Atherosclerosis Risk in Communities. Arterioscler Thromb Vasc Biol. 1997;17:1171-7.

[23] Zou Z, Xu X, Huang Y, Xiao X, Ma L, Sun T, et al. High serum level of lutein may be protective against early atherosclerosis: the Beijing atherosclerosis study. Atherosclerosis. 2011;219:789-93.

[24] Leermakers ET, Darweesh SK, Baena CP, Moreira EM, Melo van Lent D, Tielemans MJ, et al. The effects of lutein on cardiometabolic health across the life course: a systematic review and metaanalysis. Am J Clin Nutr. 2016;103:481-94.

[25] Kim JE, Leite JO, DeOgburn R, Smyth JA, Clark RM, Fernandez ML. A lutein-enriched diet prevents cholesterol accumulation and decreases oxidized LDL and inflammatory cytokines in the aorta of guinea pigs. J Nutr. 2011;141:1458-63.

[26] Han H, Cui W, Wang L, Xiong Y, Liu L, Sun X, et al. Lutein prevents high fat diet-induced atherosclerosis in ApoE-deficient mice by inhibiting NADPH oxidase and increasing PPAR expression. Lipids. 2015;50:261-73.

[27] Li SY, Fung FK, Fu ZJ, Wong D, Chan HH, Lo AC. Anti-inflammatory effects of lutein in retinal ischemic/hypoxic injury: in vivo and in vitro studies. Invest Ophthalmol Vis Sci. 2012;53:5976-84.

[28] Izumi-Nagai K, Nagai N, Ohgami K, Satofuka S, Ozawa Y, Tsubota K, et al. Macular pigment lutein is antiinflammatory in preventing choroidal neovascularization. Arterioscler Thromb Vasc Biol. 2007;27:2555-62.

[29] Tian Y, Kijlstra A, van der Veen RL, Makridaki M, Murray IJ, Berendschot TT. The effect of lutein supplementation on blood plasma levels of complement factor D, C5a and C3d. PLoS One. 2013;8:e73387.

[30] Tian Y, Kijlstra A, van der Veen RL, Makridaki M, Murray IJ, Berendschot TT. Lutein supplementation leads to decreased soluble complement membrane attack complex sC5b-9 plasma levels. Acta Ophthalmol. 2015;93:141-5.

[31] Lidebjer C, Leanderson P, Ernerudh J, Jonasson L. Low plasma levels of oxygenated carotenoids in patients with coronary artery disease. Nutr Metab Cardiovasc Dis. 2007;17:448-56.

[32] Koh WP, Yuan JM, Wang R, Lee YP, Lee BL, Yu MC, et al. Plasma carotenoids and risk of acute myocardial infarction in the Singapore Chinese Health Study. Nutr Metab Cardiovasc Dis. 2011;21:68590 . 
[33] Xu X, Wang Y, Constantinou AI, Stacewicz-Sapuntzakis M, Bowen PE, van Breemen RB. Solubilization and stabilization of carotenoids using micelles: delivery of lycopene to cells in culture. Lipids. 1999;34:1031-6.

[34] Walston J, Xue Q, Semba RD, Ferrucci L, Cappola AR, Ricks M, et al. Serum antioxidants, inflammation, and total mortality in older women. Am J Epidemiol. 2006;163:18-26.

[35] Voleti B, Agrawal A. Statins and nitric oxide reduce C-reactive protein production while inflammatory conditions persist. Mol Immunol. 2006;43:891-6.

[36] Arnaud C, Burger F, Steffens S, Veillard NR, Nguyen TH, Trono D, et al. Statins reduce interleukin-6-induced C-reactive protein in human hepatocytes: new evidence for direct antiinflammatory effects of statins. Arterioscler Thromb Vasc Biol. 2005;25:1231-6.

[37] Nilsson L, Eriksson P, Cherfan P, Jonasson L. Effects of simvastatin on proinflammatory cytokines and matrix metalloproteinases in hypercholesterolemic individuals. Inflammation. 2011;34:225-30.

[38] Wiklund O, Mattsson-Hulten L, Hurt-Camejo E, Oscarsson J. Effects of simvastatin and atorvastatin on inflammation markers in plasma. J Intern Med. 2002;251:338-47.

[39] Lyngdoh T, Vollenweider P, Waeber G, Marques-Vidal P. Association of statins with inflammatory cytokines: a population-based Colaus study. Atherosclerosis. 2011;219:253-8.

[40] Kim JH, Na HJ, Kim CK, Kim JY, Ha KS, Lee H, et al. The non-provitamin A carotenoid, lutein, inhibits NF-kappaB-dependent gene expression through redox-based regulation of the phosphatidylinositol 3-kinase/PTEN/Akt and NF-kappaB-inducing kinase pathways: role of $\mathrm{H}(2) \mathrm{O}(2)$ in NF-kappaB activation. Free Radic Biol Med. 2008;45:885-96.

[41] Krishnaswamy R, Devaraj SN, Padma VV. Lutein protects HT-29 cells against Deoxynivalenolinduced oxidative stress and apoptosis: prevention of NF-kappaB nuclear localization and down regulation of NF-kappaB and Cyclo-Oxygenase-2 expression. Free Radic Biol Med. 2010;49:50-60.

[42] Jin XH, Ohgami K, Shiratori K, Suzuki Y, Hirano T, Koyama Y, et al. Inhibitory effects of lutein on endotoxin-induced uveitis in Lewis rats. Invest Ophthalmol Vis Sci. 2006;47:2562-8. 


\section{Figure legends}

Figure 1. Intracellular lutein concentrations increased in a dose- and time-dependent manner when peripheral blood mononuclear cells from patients with stable angina were treated with lutein ex vivo. (A) Intracellular lutein concentrations after treatment with cell medium containing unloaded micelles $(0 \mu \mathrm{M})$ or micelles loaded with different concentrations of lutein $(1 \mu \mathrm{M}, 5 \mu \mathrm{M}$ or $25 \mu \mathrm{M})$ for 24 hours $(\mathrm{n}=6-16)$. ${ }^{* * *} p$ for trend $<0.001$. (B) Intracellular lutein concentrations after treatment with cell medium containing unloaded micelles (filled circles) or micelles loaded with $25 \mu \mathrm{M}$ lutein (filled squares) from baseline to 48 hours $(\mathrm{n}=6)$. ${ }^{* *} p$ for trend $<0.01$. Differences between related samples over time were analyzed by Friedman's test. Points and error bars represent mean \pm SEM.

Figure 2. Lutein treatment of LPS-stimulated peripheral blood mononuclear cells (PBMCs) decreased the release of cytokines into cell supernatants in a dose-dependent manner. PBMCs from patients with stable angina $(n=8-14)$ were treated with unloaded micelles $(0 \mu M)$ or micelles loaded with different concentrations of lutein $(1 \mu \mathrm{M}, 5 \mu \mathrm{M}$ or $25 \mu \mathrm{M})$ for 24 hours prior to addition of $1 \mathrm{ng} / \mathrm{mL}$ LPS for a further 24 hours. The percentage changes of supernatant concentrations of IL-6, IL-1 $\beta$ and TNF compared to cells treated with $0 \mu \mathrm{M}$ Lutein are shown in (A), (B) and (C), respectively. Bars represent the median percentage decrease of each cytokine with different doses of lutein in relation to unloaded micelles. Error bars represent interquartile range. * $p<0.05$ vs $0 \mu \mathrm{M}$ lutein; ** $p<0.01$ vs $0 \mu \mathrm{M}$ lutein (2-tailed Wilcoxon Signed Ranks Test).

Figure 3. Lutein treatment of LPS-stimulated peripheral blood mononuclear cells (PBMCs) reduced the gene expression of cytokines. PBMCs from patients with stable angina $(n=5)$ were treated with unloaded micelles $(0 \mu \mathrm{M})$ or micelles loaded with $25 \mu \mathrm{M}$ lutein for 24 hours before incubation with $1 \mathrm{ng} / \mathrm{mL}$ LPS for a further 4 hours. Figures represent the relative mRNA levels of $I L-6$ (A), $I L-1 \beta$ (B) and TNF (C) in each of the 5 experiments. * $p<0.05$ vs $0 \mu \mathrm{M}$ lutein (2tailed Wilcoxon Signed Ranks Test). 
Table 1. Baseline characteristics of SA and ACS patients. Data are presented as n (\%) or median (inter-quartile range).

\begin{tabular}{l|lll}
\multicolumn{1}{l}{} & $S A$ & $A C S$ & $p$-values \\
\hline Age, years & $\mathrm{n}=134$ & $\mathrm{n}=59$ & \\
Female, $n(\%)$ & $66(60-72)$ & $68(61-74)$ & 0.187 \\
Current smokers, $n(\%)$ & $18(13.4)$ & $16(27)$ & 0.285 \\
Waist circumference (cm) & $100(94-108)$ & $98(93-108)$ & 0.061 \\
Hypertension, $n(\%)$ & $72(54)$ & $29(49)$ & 0.831 \\
Type 2 diabetes, $n(\%)$ & $21(16)$ & $20(34)$ & 0.558 \\
Prior history of ACS, $n(\%)$ & $21(16)$ & $10(18)$ & 0.004 \\
Statin treatment, $n(\%)$ & $104(78)$ & $17(29)$ & 0.765 \\
IL-6 (pg/mL) & $2.21(1.45-3.03)$ & $5.01(2.68-9.36)$ & 0.000 \\
Total cholesterol, $m M$ & $3.90(3.40-4.65)$ & $5.10(4.20-6.10)$ & 0.000 \\
LDL cholesterol, $m M$ & $2.10(1.70-2.50)$ & $3.20(1.98-3.85)$ & 0.000 \\
HDL cholesterol, $m M$ & $1.20(0.97-1.40)$ & $1.20(1.00-1.50)$ & 0.370 \\
Triglycerides, $m M$ & $1.20(0.93-1.70)$ & $1.40(1.08-2.13)$ & 0.028 \\
Total carotenoids, $\mu M$ & $1.43(0.96-2.02)$ & $1.57(1.08-2.46)$ & 0.311 \\
Lutein+zeaxanthin, $\mu M$ & $0.27(0.19-0.34)$ & $0.30(0.19-0.42)$ & 0.083 \\
$\beta$-cryptoxanthin, $\mu M$ & $0.09(0.05-0.20)$ & $0.10(0.05-0.19)$ & 0.569 \\
Lycopene, $\mu M$ & $0.41(0.25-0.65)$ & $0.37(0.26-0.58)$ & 0.685 \\
$\alpha$-carotene, $\mu M$ & $0.14(0.078-0.25)$ & $0.18(0.11-0.34)$ & 0.112 \\
$\beta$-carotene, $\mu M$ & $0.33(0.20-0.58)$ & $0.35(0.21-0.68)$ & 0.732 \\
\hline & & & \\
\hline
\end{tabular}

SA, Stable angina; ACS, Acute coronary syndrome; IL-6, Interleukin-6; LDL, Low density lipoprotein; HDL, High Density Lipoprotein. 
Table 2. Cross-sectional associations between total or single carotenoids and IL-6 in plasma in SA patients, ACS patients and the whole cohort (SA + ACS). Values are given as Spearman's rank correlation coefficients.

\begin{tabular}{l|lll}
\multicolumn{2}{c}{ SA } & ACS & Whole cohort \\
\hline Total carotenoids & $-0.203^{*}$ & -0.126 & -0.125 \\
Lutein+zeaxanthin & $-0.366^{* * *}$ & -0.267 & $-0.190 *$ \\
$\beta$-cryptoxanthin & 0.017 & -0.080 & 0.022 \\
lycopene & -0.170 & -0.058 & -0.157 \\
$\alpha$-carotene & -0.184 & 0.023 & -0.053 \\
$\beta$-carotene & -0.092 & -0.036 & -0.051 \\
\hline
\end{tabular}

SA, Stable angina; ACS, Acute coronary syndrome.

$* p<0.05 ; * * * p<0.001$ 
Figure 1

A
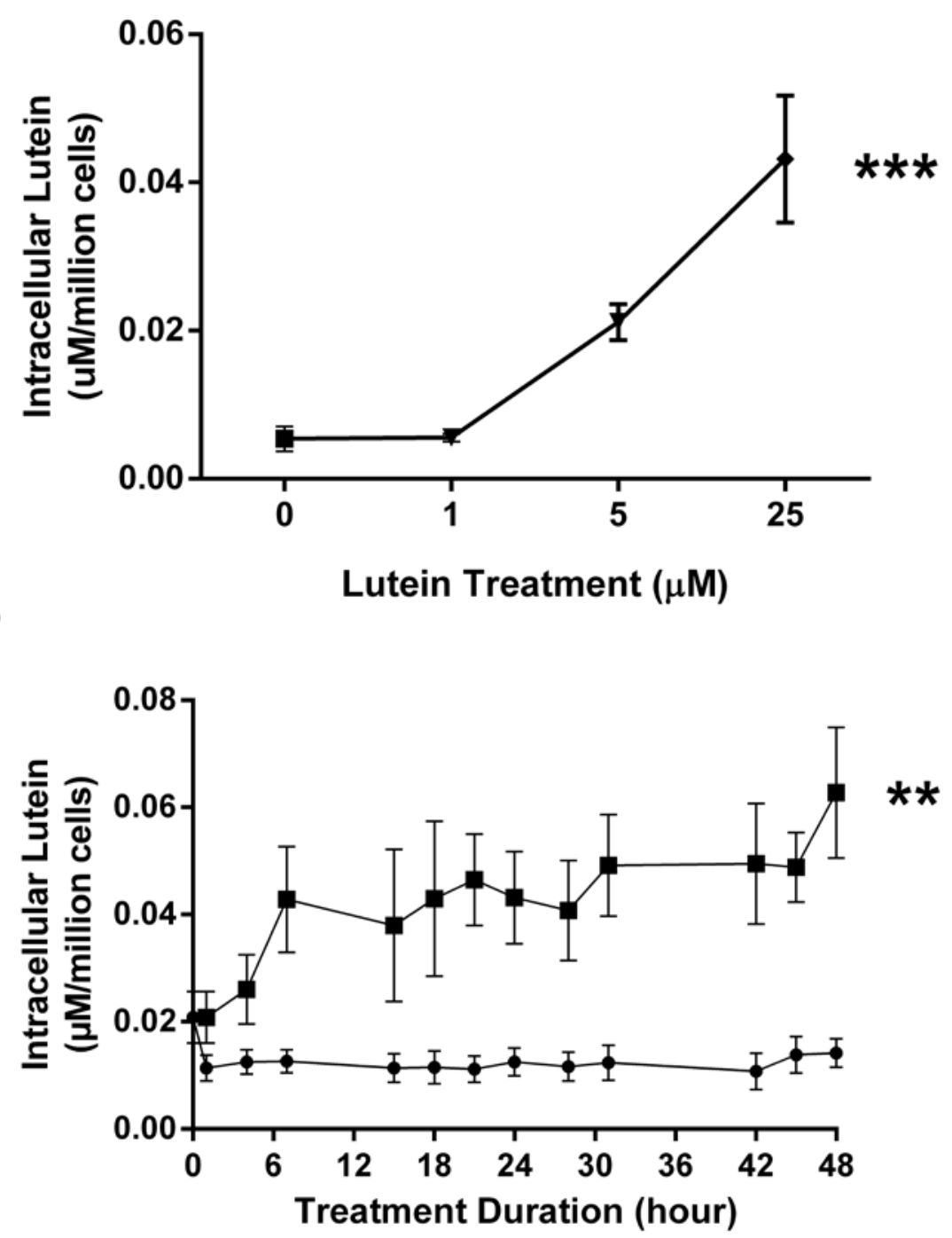
Figure 2
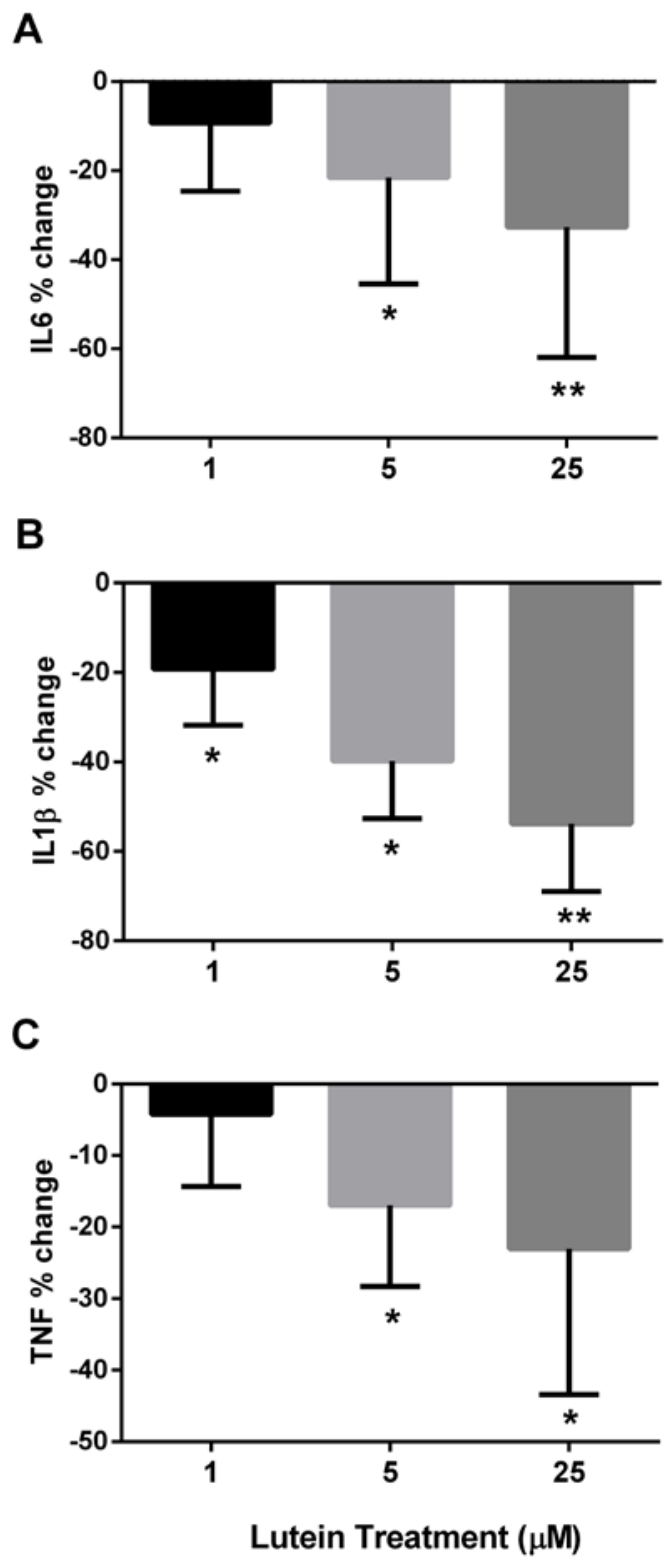
Figure 3

A

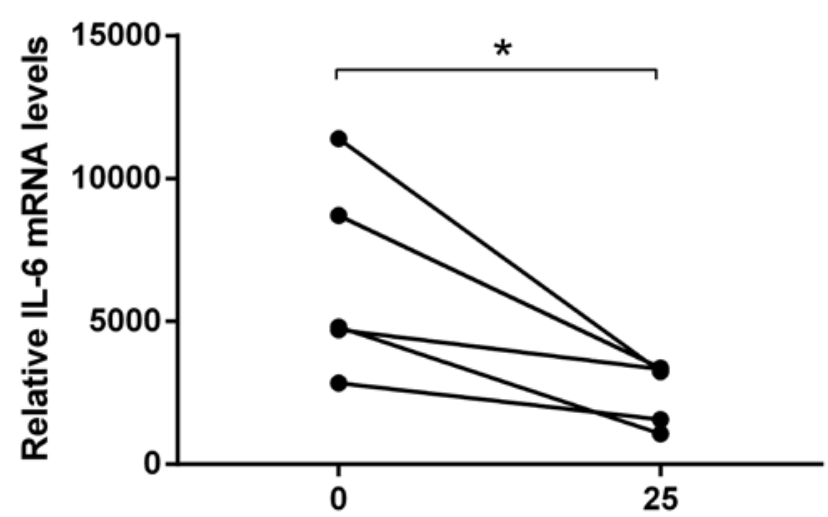

B

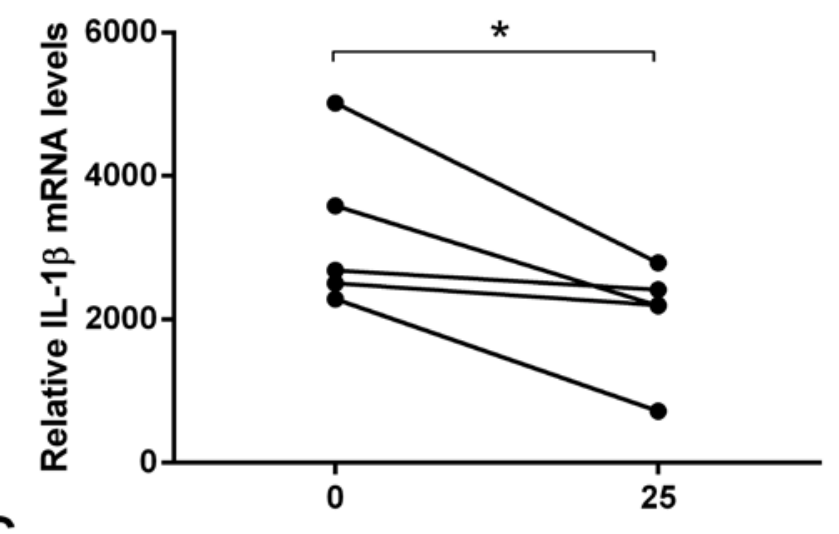

C

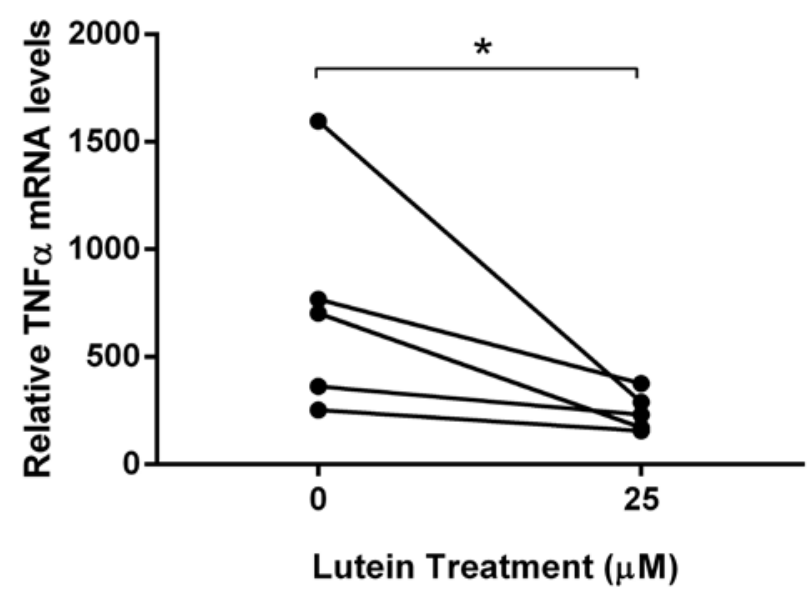

